\title{
Longitudinal study of the bone mineral content and of soft tissue composition after spinal cord section
}

\author{
E Wilmet, AA Ismail, A Heilporn, D Welraeds and P Bergmann \\ Centre de Traumatologie et de Réadaptation, Université Libre de Bruxelles Service de Médicine Nucléaire, \\ Hôpital Universitaire Brugmann, Université Libre de Bruxelles, 1020 Brussels, Belgium
}

\begin{abstract}
We present the results of a 1 year longitudinal study of bone mineral measurements and soft tissue composition in supra- and infra-lesional areas of 31 patients with a spinal cord injury (level D2-L3). Like others, we observed a rapid decrease of BMC in the paralysed areas, of $\sim 4 \% /$ month during the first year in areas rich in trabecular bone and of $\sim 2 \% /$ month in areas containing mainly compact bone. Lean soft tissue mass (muscle mass) decreases dramatically during the first months post injury in the legs, while fat content tends to increase. Though lean mass is better maintained in patients who develop spasticity, the evolution of BMC does not differ significantly between the groups of flaccid and spastic patients. In patients with partial or complete neurological recovery, a deficit in BMC of $\sim 10 \%$ with regards to the initial value is still observed at 1 year in the lower limbs. The lean mass of the upper limbs increases early after the cord injury, because of intensive rehabilitation. No significant change in BMC was observed in the supra-lesional areas. These data confirm the rapid loss of bone in the paralysed areas of paraplegic patients, which occurs independently of the presence of spontaneous muscle activity or of passive verticalisation. In patients with recovery, BMC does not return to pre-injury values within 1 year. Thus, there would be an interest in preventing bone loss early in the course of the disease.
\end{abstract}

Keywords: spinal cord injury; disuse osteoporosis; tissue composition; bone mineral

\section{Introduction}

An important degree of osteoporosis can be observed radiologically in the paralysed areas of patients with traumatic paraplegia, particularly in areas rich in trabecular bone such as the superior tibial metaphysis. ${ }^{1,2}$ Loss of bone in these patients is accompanied by an important increase in urinary calcium and hydroxyproline, two markers of an increased bone resorption, ${ }^{3}$ which peak around 6 weeks after the spinal cord section. Few longitudinal studies have been conducted in relatively small numbers of patients to quantify bone loss and to evaluate its kinetics. ${ }^{4,5,6}$ The main longitudinal study is that of Biering-Sorensen who followed bone mineral density (BMD) of the distal forearm, lumbar spine, femur and proximal tibia in eight patients with a spinal cord injury using DPA. ${ }^{3}$ Their group included tetra (two)- and paraplegic (six) patients. At inclusion, the injury dated from less than 1 month to more than 5 months. The more rapid loss was observed at the upper tibia, which is rich in trabecular bone, with

Correspondence: E Wilmet, Centre de Traumatologie et de Réadaptation (Université Libre de Bruxelles), 4 Place Van Gehuchten 1020 Brussels, Belgium. a total loss of $\sim 50 \%$ attained at 18 months, followed by a new steady state. In the femoral shaft, the loss was more linear; it amounted to $\sim 20 \%$ at 18 months, and continued afterwards. No loss was observed in the lumbar spine, nor in the arms. More recently, Garland et $a l^{5}$ published a transverse/longitudinal study in which 25 male acute-spinal cord injured patients were included $3 \frac{1}{2}$ months after their injury and compared with 10 normal subjects and 20 chronic spinal cord injured patients studied more than 5 years after their accident. Twelve of the 25 acute patients were re-measured $1 \frac{1}{2}$ year after their injury. Tetra- and paraplegic patients were included in their groups. The bone mineral content was measured by DPA in the head, arms, trunk, pelvis and legs. The authors also report on the bone mineral density of the distal femur. They show bone loss in all the areas studied, except the trunk and the head, in acute-spinal cord injured patients. Bone mineral was stable in the arms thereafter, but it took $1 \frac{1}{2}$ year to attain a new steady state in the pelvis and legs. In the arms, there was a significant difference in bone mineral content (BMC) between tetra- and paraplegic patients, but stability is observed after the acute period in both subgroups. On the other hand, there are few data on body composition after spinal injury. The main 
conclusion of these studies ${ }^{6-8}$ is a general increase in fat content.

In this study, we took advantage of the new DXA technology to measure sequentially in spinal cord injury patients both total body and regional bone mineral (TBBM) and the lean and fat contents of body regions located in the paralysed and unharmed areas, during the first year following the injury. Only paraplegic patients with a complete lesion were included in the global analysis, although some patients undergoing partial or total neurological recovery were compared to this group. As it was shown that the loss occurs early, the first TBBM measurement was realised within 8 weeks post-injury at the latest (1 to 8 weeks).

\section{Methods}

\section{Patients}

A consecutive series of 31 patients (seven female and 24 male) presenting at the Centre de Traumatologie et de Revalidation within 8 weeks of a traumatic spinal cord injury were recruited for this study. Each patient gave informed consent. The mean age of the group was 32.5 years (range: 17.5-65.5) and the lesion level was T2 to L3 (no tetraplegic patients included). The first TBBM measurement was performed around 5 weeks $(n=31)$. Then, measurements were performed around 10 weeks $(n=26), 20$ weeks $(n=16), 30$ weeks $(n=11), 40$ weeks $(n=4)$ and 50 weeks $(n=2)$. Soft tissue composition could not be calculated in one patient (no soft tissue bar). Three leg measurements could not be taken into account at 10 weeks and two at 20 weeks because of an artefact. $\mathrm{A}+$ or ++ degree of spasticity (estimated by the clinician) developed in 15 patients. Six patients recovered walking with an orthosis at the end of this study, and two others assumed a standing position. None of the patients developed clinical or radiological periarticular ossifications.

\section{Technique}

Total body and regional bone mineral were measured by DXA using a Hologic W2000 apparatus. A soft tissue phantom was measured together with the patient in order to calculate the soft tissue composition. The software used to calculate bone mineral contents and tissue composition are those of the constructor. Long term imprecision of TBBM and of fat content with this device is given as $1 \%$. Only areas in which the result could not be altered by the presence of synthesis material were analysed. This excludes most often the trunk, sometimes a broken limb. The results of bone measurements are given in BMC $(\mathrm{g})$, or are expressed as a percentage of their initial values in order to make pooling of the results possible. The results of the fat content and of lean tissue mass are given in $\mathrm{g}$ or in $\%$ of the initial value. Linear and non-linear regression were used to calculate rates of variation of BMC or soft tissues.

\section{Results}

\section{Bone mineral content}

(1) Patients with a complete lesion showing no trend towards recovery $(n=25)$ The trunk and lumbar spine were excluded from the analysis because they were too often the place of an osteosynthesis.

The area where bone loss was the fastest was the pelvis (Figure 1A), with a loss of 40 to $45 \%$ of the initial value after one year. In the lower limbs taken as a whole, the loss was linear and attained only $25 \%$ at the end of the first year (Figure 1B). As observed by Biering-Sorensen ${ }^{3}$ in the upper tibia, which is also rich in trabecular bone, the loss in the pelvic area was non-linear and tended to level-off already at 1 year. In the lower limbs however, the loss is steadily continuing at the end of the first year. The bone mineral content in the arms does not vary significantly during the same period. In the skull, the BMC first decreases by $\sim 10 \%$, and then increases again from the 4th to the 12th month.

(2) Patients with a partial recovery $(n=6)$ These patients, who have been fully paralysed for some weeks after their trauma, lose bone during this period in the paralysed areas, and do not recover bone mass in these areas during the observation period, notwithstanding the motor recovery and assuming a standing position. At the end of 1 year, bone loss is nevertheless less than occurs in the paraplegic patients who do not recover, being as a mean of $30 \%$ in the pelvis and of $10 \%$ in the lower limbs (Figure 2).

(3) Comparison of flaccid $(n=12)$ and spastic $(n=11)$ paraplegia $\mathrm{BMC}$ of the paralysed areas varies in a very similar way in the lower limbs of spastic and flaccid patients (Figure 3).
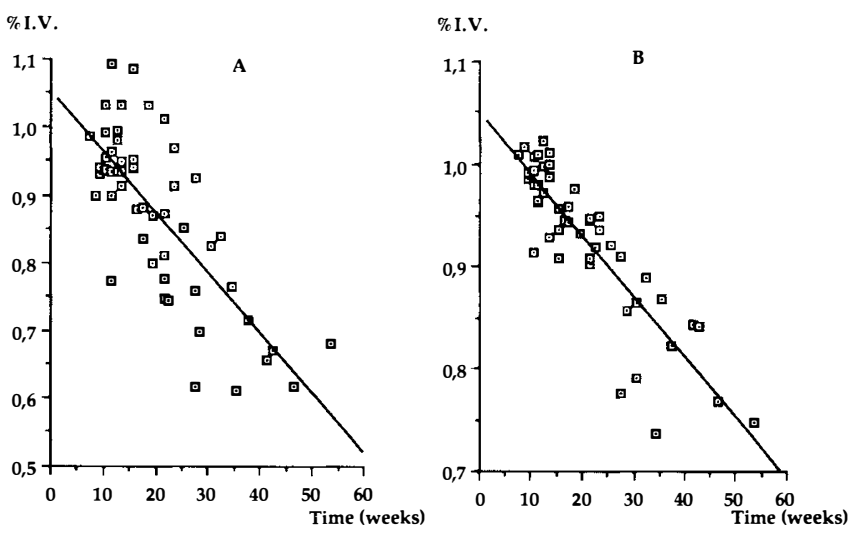

Figure 1 Evolution in BMC in the pelvis (A) and legs (B) of paraplegic patients who do not recover walking. The evolution of BMC with time (weeks) is described by the relations: pelvis: $\mathrm{BMC}=1.03-0.0089 \times(r=0.765)$; legs: $\mathrm{BMC}=1.03-0.0059 \times(r=0.879)$ 
676

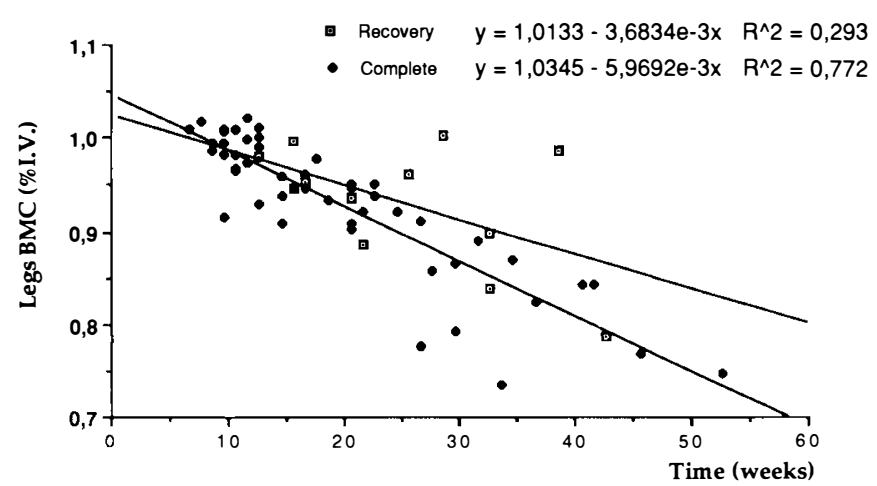

Figure 2 Evolution of BMC in patients with no recovery as compared with those who recover walking

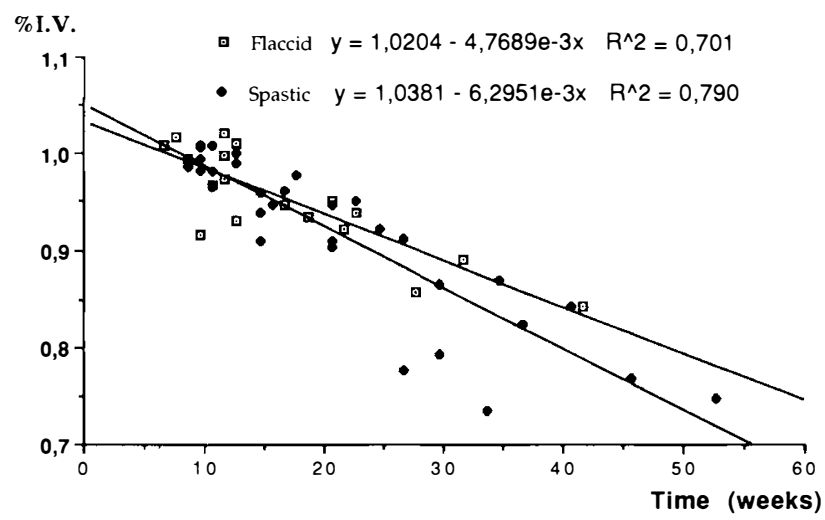

Figure 3 Evolution of BMC in spastic versus flaccid paraplegic patients

\section{Regional lean mass}

In the legs, the lean mass was decreased by $\sim 15 \%$ after 1 year. This decrease occurred rapidly after the spinal injury (15 weeks). The decrease of lean mass was different between the spastic and flaccid groups; as a mean, spastic patients did not lose lean mass at all in their paralysed areas (Figure 4).

In the arms, as a result of active rehabilitation, lean mass increased by $30 \%$ after 6 months. At 1 year, it stabilised, being about $15 \%$ higher than its initial value (Figure 5).

\section{Regional fat mass}

A large dispersion of the individual evolutions prevents any general overview of the evolution of the fat content. There is clearly a trend for the fat content to increase in the lower limbs and to decrease in the upper limbs of some of the patients, but this variation can be obscured by the patients' weight, which affects more generally the fat content.

\section{Discussion}

Loss of bone in the paralysed areas is a well known complication of spinal cord injury. ${ }^{1-5}$ It is not harmless,

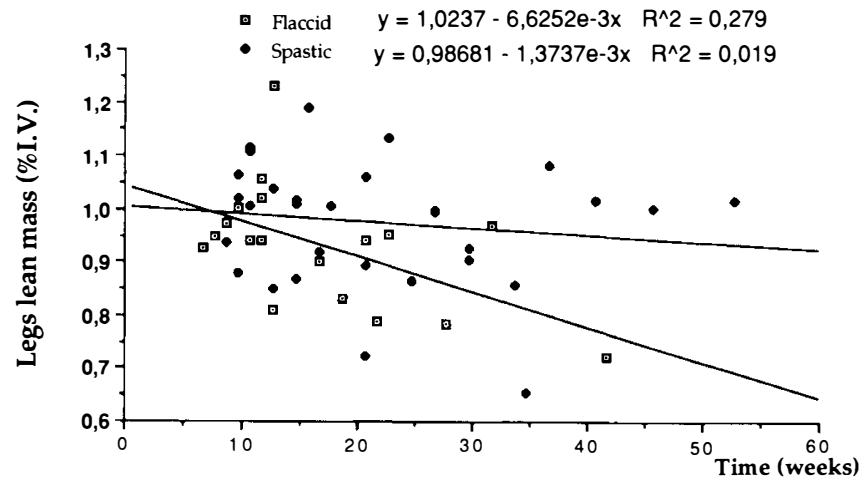

Figure 4 Leg lean mass in spastic and flaccid paraplegic patients

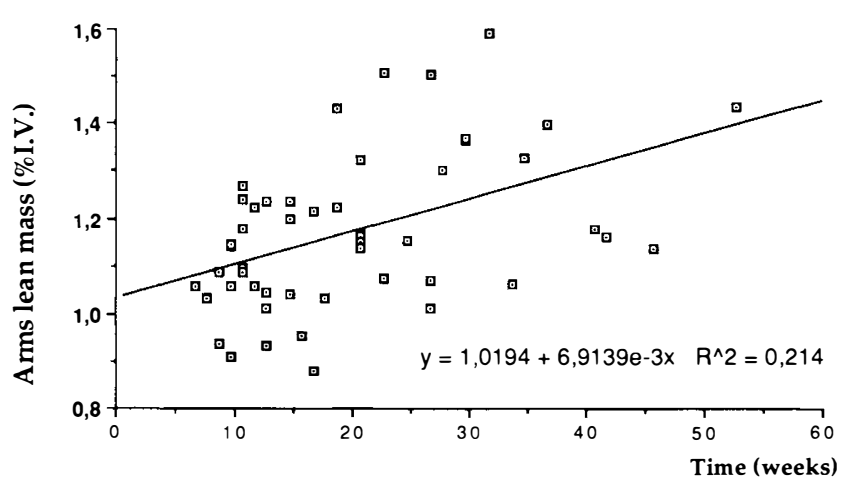

Figure 5 Evolution of arms lean mass during the first year of paraplegia

since it will make bones fragile and increase the fracture risk in this population. Fractures are indeed a frequent event in paraplegic patients undergoing modern rehabilitation programs, ${ }^{9}$ and their treatment may present unusual difficulties. ${ }^{10}$ The aim of our longitudinal study was to describe the natural history of bone loss in a population of paraplegic patients. Our results are confirmatory that an appreciable amount of bone is lost rapidly in the paralysed areas after the spinal cord injury.

Evolution of the lean mass is what is to be expected: a dramatic decrease in the paralysed areas, reflecting muscle waste, in flaccid patients; a relative conservation in patients with spasticity; and a variable increase in the upper extremities, depending on the compliance with the rehabilitation program.

The loss of bone in the paralysed areas occurs more rapidly in areas rich in trabecular bone, such as the pelvis, but it continues more steadily in cortical bone, so that the final result could in the end be approximately the same ( $\sim 50 \%$ loss in the long term).${ }^{4}$ Our study adds some information concerning subgroups of paraplegic patients: (1) though the muscular mass is more conserved in patients with spasticity than in those who are flaccid, the loss of bone in the paralysed areas 
does not significantly differ between these groups. This shows that simple conservation of muscle mass is not sufficient to maintain skeletal integrity, in the absence of normal motion; (2) patients who recover a near normal mobility still lose bone at 1 year. Thus, in patients who are likely to recover, a programme of prevention of bone loss should be instituted early in the course of the disease.

The evolution of BMC in the non-paralysed areas remain controversial: some authors ${ }^{4}$ did not find bone loss in the upper extremities of their patients while Garland ${ }^{5}$ observed an initial decrease of BMC in the supra-lesional areas, followed by a partial recovery. The evolution of the skull BMC of our patients would be similar to this, but with complete recovery. In the arms, patients did not lose any bone mineral. Differences between studies with regard to the evolution of bone mineral in the non-paralysed areas could result from the intensity and precocity of the rehabilitation program, and from differences in the patient's population, with patients more severely injured and in worse general condition losing bone, the others not. It is noteworthy that our patients did not have a significant bone gain in the upper extremities, in front of a significant increase of muscular mass and activity. Probably an effect of exercising on bone mass will take longer than the period of intensive rehabilitation. After this period, patients are discharged and lose at least in part the muscle they had gained.

\section{Acknowledgement}

P Bergmann is the recipient of a grant from the Fonds de la Recherche Scientifique Médicale Belge (no. 9.4544.92).

\section{References}

1 Chantraine A. Actual concept of osteoporosis in paraplegia. Paraplegia 1978; 16: 51-58.

2 Biering-Sorensen F, Bohr H, Schaadt OP. Bone mineral content of the lumbar spine and lower extremities years after spinal cord lesion. Paraplegia 1988; 26: 293-301.

3 Bergmann $\mathrm{P}$ et al. A longitudinal study of calcium and bone metabolism in paraplegic patients. Paraplegia 1977-1978; 15: $147-159$.

4 Biering-Sorensen F, Bohr H, Schadt OP. Longitudinal study of bone mineral content in the lumbar spine, the forearm and the lower extremities after spinal cord injury. Europ J Clin Invest 1990; 20: 330-335.

5 Garland DE et al. Osteoporosis after spinal cord injury. J Orthop Res 1992; 10: 371-378.

6 Sedlock DA, Laventure SJ. Body composition and resting energy expenditure in long term spinal cord injury. Paraplegia 1990; 28: 448-454.

7 Bulbulian $\mathrm{R}$ et al. Body composition in paraplegic male atheletes. Med Sci Sports Exercise 1987; 19: 195-201.

8 Lussier L et al. Body composition comparison in two elite female wheelchair athletes. Paraplegia 1983; 21: 16-22.

9 Eichenholtz SN. Management of long bone fractures in paraplegic patients. J Bone Joint Surg 1963; 45A: 299-310.

10 Sobel M, Lyden JP. Long bone fracture in a spinal-cordinjured patient: complication of treatment - A case report and review of the literature. J Trauma 1991; 31: 1440-1444. 